\title{
Generalizing the restricted three-body problem. The Bianular and Tricircular coherent problems
}

\author{
F. Gabern and À. Jorba
}

\author{
Departament de Matemàtica Aplicada i Anàlisi, Universitat de Barcelona, Gran Via 585, 08007 Barcelona, Spain \\ e-mail: angel@maia.ub.es
}

Received 3 December 2003 / Accepted 4 March 2004

\begin{abstract}
In this paper we construct two models for the motion of a particle under the gravitational attraction of Sun, Jupiter, Saturn and Uranus, that can be seen as a generalization of the well known Restricted Three-Body Problem (RTBP). Both models are obtained by computing quasi-periodic solutions - with two basic frequencies - of a suitable $N$-body problem. The first model is based on a quasi-periodic solution of the planar Sun-Jupiter-Saturn Three-Body problem, that tries to approach the real motion of Jupiter. The second model is based on a quasi-periodic solution of the Sun-Jupiter-Saturn-Uranus Four-Body problem. In both cases, we derive the equations of motion for a particle under the gravitational attraction of these bodies as a quasi-periodic time-dependent perturbation of the well-known RTBP.
\end{abstract}

Key words. celestial mechanics - methods: $N$-body simulations - methods: numerical

\section{Introduction}

The Restricted Three Body Problem (RTBP) has been widely used to model the dynamics of an asteroid under the attraction of two massive bodies. A typical example are the so-called Trojan asteroids, located near the triangular points of the SunJupiter system. However, it is clear that the RTBP is not useful for accurate computations since it does not include many important effects, like the eccentricity of Jupiter and the presence of other planets (for instance, see Gabern et al. 2003).

The usual simulations for the motion of an asteroid are based on numerical integrations of a suitable $N$-body problem. For instance, for the Trojan asteroids it is quite common to integrate numerically the Outer Solar System plus the asteroid (see Schubart \& Bien 1986; Milani 1993; Levison et al. 1997; Pilat-Lohinger et al. 1999; Tsiganis et al. 2000), although it is possible to use more general models, see Robutel \& Laskar (2001).

In Gabern \& Jorba (2001), a model for the motion of an asteroid under the gravitational attraction of Sun, Jupiter and Saturn was developed and studied. This model was based on computing, in suitable coordinates, a periodic orbit for these three bodies. This model was the natural extension of the RTBP to include three primaries, in the sense that they move in the simplest solution that resembles the planetary system. Then, assuming that Sun, Jupiter and Saturn move on this orbit, it was not difficult to write the equations of motion for the asteroid; this is what we refer as BCCP. In suitable coordinates,

Send offprint requests to: F. Gabern, e-mail: gabern@mat.ub.es the $\mathrm{BCCP}$ is a periodic time-dependent perturbation of the well known RTBP.

In this paper we derive two analytical models for the motion of a particle in the Solar system, that can be seen as extensions of the RTBP. The first of them is called the Bianular problem (BAP) and tries to account for the effect of Sun, Jupiter and Saturn. The second model is called Tricircular Coherent Problem (TCCP) and it includes Sun, Jupiter, Saturn and Uranus. Both models are based on the computation of a true quasi-periodic solution, with two basic frequencies, of the planar $N$-body problem for Sun and the considered planets. Then, the equations of motion for the particle are written in a suitable reference frame, such that the resulting model can be seen as a quasi-periodic time-dependent perturbation of the RTBP. Our models can also be seen as natural improvements of the Elliptic RTBP (Szebehely 1967), the Bicircular Problem (Cronin et al. 1964) and the BCCP (Gabern \& Jorba 2001).

These models are still much closer to the RTBP than to the numerical simulations based on the Outer Solar System. However, we believe that they are a first step in the understanding, by means of analytical techniques, of the real dynamics of the asteroids. For instance, due to the explicit character of our models, it is possible to apply semi-analytical methods (like normal forms computations, see Jorba 1999) to derive stability results for a region of the phase space (in the same way it has been done in the RTBP, see Giorgilli et al. 1989; Simó 1989; Celletti \& Giorgilli 1991; Giorgilli \& Skokos 1997; Skokos \& Dokoumetzidis 2000) or to describe the phase space near the collinear points of the RTBP (see Jorba \& Masdemont 1999). 


\subsection{The Bianular problem}

It is based on the computation of a 2-D invariant torus of the planar Sun-Jupiter-Saturn Three-Body Problem. To this end, we reduce the Hamiltonian of the planar TBP written in the Jacobi coordinates in a uniformly rotating reference frame from 4 to 3 degrees of freedom via a canonical change of variables that uses the angular momentum first integral. Then, we use the method developed in Castellà \& Jorba (2000) in order to compute a quasi-periodic solution (with two basic frequencies) of the reduced equations.

Due to the Hamiltonian character of the problem, invariant tori are generically not isolated. In fact, an invariant torus with $r$ basic frequencies belongs to a $r$-dimensional (Cantor) family of $r$-dimensional invariant tori (for mode details see, for instance, Jorba \& Villanueva 1997). Thus, we can "move" inside this family to look for an invariant torus for which the motion of Jupiter has a prescribed characteristic. We use a continuation method (using the angular momentum as a parameter) to find a quasi-periodic solution of the reduced problem for which Jupiter's osculating eccentricity is the actual one. Then, it is not difficult to write this 2-D invariant torus in the non-reduced coordinates.

Finally, the equations of motion of a massless particle that moves under the attraction of these three main bodies (supposing that they move in the previously computed quasi-periodic solution) are easily derived. This is a restricted four body problem that we call the Bianular Problem (BAP).

\subsection{The Tricircular coherent problem}

It is based on the computation of a quasi-periodic solution (with two basic frequencies) of the planar Sun-Jupiter-Saturn-Uranus Four-Body Problem. The model is derived as follows. As before, we use the angular momentum to reduce the Four-Body problem (written in the Jacobi coordinates) from 6 to 5 degrees of freedom. Then, we compute a quasi-periodic solution (with two basic frequencies) for the Four-Body Problem, in which the mean motions of Jupiter, Saturn and Uranus are adjusted to the actual values. Finally, a Restricted Five-Body Problem (that we call Tricircular Coherent Problem, or TCCP) can be constructed by writing the equations of a particle moving under the influence of the four bodies. Note that this solution of the Sun-Jupiter-Saturn-Uranus system is the simplest planetary one. In this sense, the TCCP is the natural extension of the RTBP to include four primaries.

\section{The planar N-planetary problem}

The planar $N$-planetary problem is a planar problem of $N+1$ bodies in which one of the masses is much bigger and the remaining $N$ bodies move around the biggest mass in nearly Keplerian orbits.

\subsection{Rotating Jacobi coordinates}

Here we describe the generalized Jacobi formulation for the $N$-planetary problem. Let $\mathcal{P}_{0}$ denote the biggest body (i.e., the
Sun), $\mathcal{P}_{i}, i=1, \ldots, N$, the planets, and let $m_{i}$ be the respective masses, $i=0, \ldots, N$. Then, we refer every planetary body $\mathcal{P}_{i}$ to the barycenter $B_{i-1}$ of the $i$ preceding bodies $\mathcal{P}_{0}, \ldots, \mathcal{P}_{i-1}$. Thus, we work with $N$ position vectors $\boldsymbol{q}_{i}, i=1, \ldots, N$, each one going from the barycenter $B_{i-1}$ to the body $\mathcal{P}_{i}$. Obviously, the vector $\boldsymbol{q}_{N}$ contains the barycentre of the system. In this way, we can easily have a Hamiltonian formulation of the problem that includes the reduction of the linear momentum first integral.

A description of different coordinates for $N$-planetary problems can be found in Laskar (1989), including its Hamiltonian in Jacobi coordinates,

$H=\sum_{i=1}^{N} \frac{1}{2 \alpha_{i}}\left\|\boldsymbol{p}_{i}\right\|^{2}-\sum_{0 \leq i<j \leq N} G \frac{m_{i} m_{j}}{\left\|\boldsymbol{r}_{i j}\right\|}$,

where $\alpha_{i}=\frac{m_{i} \eta_{i-1}}{\eta_{i}}, \eta_{i}=\sum_{j=0}^{i} m_{j}, \boldsymbol{p}_{i}=\alpha_{i} \dot{\boldsymbol{q}}_{i}$ and the distances between the bodies are given by the modulus of the vectors

$\boldsymbol{r}_{0 i}=\boldsymbol{q}_{i}+\sum_{j=1}^{i-1} \frac{m_{j}}{\eta_{j}} \boldsymbol{q}_{j}, \quad i=1, \ldots, N$

$\boldsymbol{r}_{i j}=\boldsymbol{q}_{j}+\sum_{k=1}^{j-1} \frac{m_{k}}{\eta_{k}} \boldsymbol{q}_{k}-\frac{\eta_{i-1}}{\eta_{i}} \boldsymbol{q}_{i}, \quad 0<i<j \leq N$.

Next, we implement a uniform counterclockwise rotation of frequency 1 with the origin at the center of masses, $\boldsymbol{q}_{i}=\mathcal{R}(t) \boldsymbol{Q}_{i}$, for $i=1, \ldots, N$, where

$\mathcal{R}(t)=\left(\begin{array}{cc}\cos (t) & -\sin (t) \\ \sin (t) & \cos (t)\end{array}\right)$

Defining new momenta as usual, $P_{i}^{(x)}=\alpha_{i}\left(\dot{Q}_{i}^{(x)}-Q_{i}^{(y)}\right)$ and $P_{i}^{(y)}=\alpha_{i}\left(\dot{Q}_{i}^{(y)}+Q_{i}^{(x)}\right)$, where $\boldsymbol{Q}_{i}=\left(Q_{i}^{(x)}, Q_{i}^{(y)}\right), \boldsymbol{P}_{i}=\left(P_{i}^{(x)}, P_{i}^{(y)}\right)$, and renaming the new variables $(\boldsymbol{Q}, \boldsymbol{P})$ as $(\boldsymbol{q}, \boldsymbol{p})$, we can write the Hamiltonian of the $N$-planetary problem in a uniformly rotating reference frame as:

$H=\sum_{i=1}^{N} \frac{\left\|\boldsymbol{p}_{i}\right\|^{2}}{2 \alpha_{i}}+\sum_{i=1}^{N}\left(q_{i}^{(y)} p_{i}^{(x)}-q_{i}^{(x)} p_{i}^{(y)}\right)-\sum_{0 \leq i<j \leq N} G \frac{m_{i} m_{j}}{\left\|\boldsymbol{r}_{i j}\right\|}$

where the distances between the bodies, $\left\|\boldsymbol{r}_{i j}\right\|$, are given by the Euclidean norm of the vectors (2).

\subsection{Reduction of one degree of freedom}

Now, we apply a canonical change of variables to reduce the Hamiltonian from $2 N$ to $2 N-1$ degrees of freedom. The idea is very well known (see, for example, Whittaker 1952) and consists of making use of the classical first integral of the angular momentum. The change of variables that we perform can be written as

$\boldsymbol{q}_{i}=\frac{\partial G}{\partial \boldsymbol{p}_{i}}, \quad \boldsymbol{P}_{i}=\frac{\partial G}{\partial \boldsymbol{Q}_{i}}, \quad i=1, \ldots, N$, 
where $\left(Q_{i}, P_{i}\right)_{i}$ are the new variables and $G(Q, p)$ is the following generating function:

$$
\begin{aligned}
G(Q, p)= & Q_{1}^{(x)}\left(p_{1}^{(x)} \cos Q_{1}^{(y)}+p_{1}^{(y)} \sin Q_{1}^{(y)}\right) \\
& -\sum_{i=2}^{N}\left[p_{i}^{(x)}\left(Q_{i}^{(x)} \cos Q_{1}^{(y)}+Q_{i}^{(y)} \sin Q_{1}^{(y)}\right)\right. \\
& \left.\quad+p_{i}^{(y)}\left(Q_{i}^{(x)} \sin Q_{1}^{(y)}-Q_{i}^{(y)} \cos Q_{1}^{(y)}\right)\right] .
\end{aligned}
$$

Note that $Q_{1}^{(x)}$ is the distance between the bodies $P_{0}$ and $P_{1}$, $Q_{i}^{(x)}$ and $Q_{i}^{(y)}$ for $i=2, \ldots, N$, are, respectively, the projections of the vector $\boldsymbol{q}_{i}$ into the $P_{0} P_{1}$ direction and its orthogonal one. $Q_{1}^{(y)}$ is the angle between the vector $\boldsymbol{q}_{1}$ and the "old" $x$ axis. $P_{i}^{(x)}, i=1, \ldots, N$, are the projections of $\boldsymbol{p}_{i}$ into the $P_{0} P_{1}$ direction and $P_{i}^{(y)}, i=2, \ldots, N$ into the orthogonal one. Finally, $P_{1}^{(y)}$ is the angular momentum of the system.

In these variables, the Hamiltonian (3) becomes independent from $Q_{1}^{(y)}$, so $P_{1}^{(y)}$ is a first integral of the system. Its value $P_{1}^{(y)}=K$ is the angular momentum. The equation of motion corresponding to $Q_{1}^{(y)}$ can be easily integrated once the remaining variables of the system have been solved. Thus, skipping the equations corresponding to $Q_{1}^{(y)}$ and $P_{1}^{(y)}$ we obtain the following $2 N-1$ degrees of freedom Hamiltonian,

$H=\frac{1}{2 \alpha_{1}}\left(\left(P_{1}^{(x)}\right)^{2}+\frac{A^{2}}{\left(Q_{1}^{(x)}\right)^{2}}\right)-K+\sum_{i=2}^{N} \frac{\left\|\boldsymbol{P}_{i}\right\|^{2}}{2 \alpha_{i}}-\sum_{i<j} G \frac{m_{i} m_{j}}{\left\|\boldsymbol{r}_{i j}\right\|}$,

where $A=\sum_{i=2}^{N}\left(Q_{i}^{(x)} P_{i}^{(y)}-Q_{i}^{(y)} P_{i}^{(x)}\right)+K$ and

$\left\|\boldsymbol{r}_{01}\right\|^{2}=\left(Q_{1}^{(x)}\right)^{2}$,

$$
\begin{aligned}
\left\|\boldsymbol{r}_{0 i}\right\|^{2}= & \left(Q_{i}^{(x)}-\frac{m_{1}}{\eta_{1}} Q_{1}^{(x)}\right)^{2}+\left(Q_{i}^{(y)}\right)^{2}-2 \sum_{j=2}^{i-1} \frac{m_{1} m_{j}}{\eta_{1} \eta_{j}} Q_{1}^{(x)} Q_{j}^{(x)} \\
& +\sum_{k, j=2}^{i-1} \frac{m_{k} m_{j}}{\eta_{k} \eta_{j}} \boldsymbol{Q}_{k} \cdot \boldsymbol{Q}_{j}+2 \sum_{j=2}^{i-1} \frac{m_{j}}{\eta_{j}} \boldsymbol{Q}_{i} \cdot \boldsymbol{Q}_{j} \quad i>1,
\end{aligned}
$$

$\left\|\boldsymbol{r}_{1 j}\right\|^{2}=\left(Q_{j}^{(x)}-\frac{\eta_{0}}{\eta_{1}} Q_{1}^{(x)}\right)^{2}+\left(Q_{j}^{(y)}\right)^{2}-2 \sum_{k=2}^{j-1} \frac{m_{k} \eta_{0}}{\eta_{1} \eta_{k}} Q_{1}^{(x)} Q_{k}^{(x)}$

$$
+\sum_{k, l=2}^{j-1} \frac{m_{k} m_{l}}{\eta_{k} \eta_{l}} \boldsymbol{Q}_{k} \cdot \boldsymbol{Q}_{l}+2 \sum_{k=2}^{j-1} \frac{m_{k}}{\eta_{k}} \boldsymbol{Q}_{j} \cdot \boldsymbol{Q}_{k} \quad j>1,
$$

$$
\begin{aligned}
\left\|\boldsymbol{r}_{i j}\right\|^{2}= & \left\|\boldsymbol{Q}_{j}-\frac{\eta_{i-1}}{\eta_{i}} \boldsymbol{Q}_{1}\right\|^{2}-2 \sum_{k=i+1}^{j-1} \frac{m_{k} \eta_{i-1}}{\eta_{k} \eta_{i}} \boldsymbol{Q}_{i} \cdot \boldsymbol{Q}_{k} \\
& +\sum_{k, l=i+1}^{j-1} \frac{m_{k} m_{l}}{\eta_{k} \eta_{l}} \boldsymbol{Q}_{k} \cdot \boldsymbol{Q}_{l}+2 \sum_{k=i+1}^{j-1} \frac{m_{k}}{\eta_{k}} \boldsymbol{Q}_{j} \cdot \boldsymbol{Q}_{k} \quad 1<i<j .
\end{aligned}
$$

\subsection{Equations of motion for $N=2$ and $N=3$}

Now we select units of distance, time and mass such that the Sun-Jupiter distance is 1 , the initial osculating period of Jupiter around the Sun is $2 \pi$, and that the total mass of Sun and Jupiter is 1 . With these units, the gravitational constant $G$ is also 1 . In these adimensional units, the masses of the bodies involved in the two and three planetary problems take the values: $m_{1}=m_{\text {jup }}=\mu=9.5387536 \times 10^{-4}\left(m_{0}=1-\mu\right.$, for Sun), $m_{2}=m_{\text {sat }}=2.855150174 \times 10^{-4}$ and $m_{3}=m_{\text {ura }}=$ $4.361228581 \times 10^{-5}$.

We also rename the coordinates as $x_{1}=Q_{1}^{(x)}, y_{1}=P_{1}^{(x)}$, and $x_{2 k-2}=Q_{k}^{(x)}, x_{2 k-1}=Q_{k}^{(y)}, y_{2 k-2}=P_{k}^{(x)}, y_{2 k-1}=P_{k}^{(y)}$ for $k=2, \ldots, N$.

\subsubsection{The SJS planar problem}

The reduced Sun-Jupiter-Saturn planar problem is a three degrees of freedom Hamiltonian system and depends on the variables $x_{1}, x_{2}, x_{3}, y_{1}, y_{2}, y_{3}$ and on the parameter angular momentum $K$ :

$$
\begin{aligned}
H(x, y, K)= & \frac{1}{2 \alpha}\left(y_{1}^{2}+\frac{A^{2}}{x_{1}^{2}}\right)+\frac{y_{2}^{2}+y_{3}^{2}}{2 \beta}-K \\
& -\frac{\alpha}{x_{1}}-\frac{(1-\mu) m_{\mathrm{sat}}}{r_{13}}-\frac{\mu m_{\mathrm{sat}}}{r_{23}}
\end{aligned}
$$

where

$r_{13}^{2}=\left(\mu x_{1}-x_{2}\right)^{2}+x_{3}^{2}$,

$r_{23}^{2}=\left((1-\mu) x_{1}+x_{2}\right)^{2}+x_{3}^{2}$,

$A=\left(x_{2} y_{3}-x_{3} y_{2}\right)+K$,

and recall that $\alpha=\mu(1-\mu)$ and $\beta=m_{\text {sat }} /\left(1+m_{\mathrm{sat}}\right)$.

\subsubsection{The SJSU planar problem}

We can also write the vector field of the reduced planar four body Sun-Jupiter-Saturn-Uranus problem depending on ten variables, $\left(x_{1}, \ldots, x_{5}, y_{1}, \ldots, y_{5}\right)$ and on the angular momentum $K$. This is a five degrees of freedom Hamiltonian system,

$$
\begin{aligned}
H(x, y, K)= & \frac{1}{2 \alpha}\left(y_{1}^{2}+\frac{A^{2}}{x_{1}^{2}}\right)+\frac{y_{2}^{2}+y_{3}^{2}}{2 \beta}+\frac{y_{4}^{2}+y_{5}^{2}}{2 \gamma}-K \\
& -\frac{\alpha}{x_{1}}-m_{\text {sat }}\left(\frac{(1-\mu)}{r_{13}}+\frac{\mu}{r_{23}}\right) \\
& -m_{\text {ura }}\left(\frac{(1-\mu)}{r_{14}}+\frac{\mu}{r_{24}}+\frac{m_{\text {sat }}}{r_{34}}\right)
\end{aligned}
$$

where

$$
\begin{aligned}
& r_{13}^{2}=\left(x_{2}-\mu x_{1}\right)^{2}+x_{3}^{2}, \\
& r_{23}^{2}=\left(x_{2}+(1-\mu) x_{1}\right)^{2}+x_{3}^{2}, \\
& r_{14}^{2}=\left(x_{5}+\beta x_{3}\right)^{2}+\left(x_{4}+\beta x_{2}-\mu x_{1}\right)^{2}, \\
& r_{24}^{2}=\left(x_{5}+\beta x_{3}\right)^{2}+\left(x_{4}+\beta x_{2}+(1-\mu) x_{1}\right)^{2}, \\
& r_{34}^{2}=\left(x_{5}-(1-\beta) x_{3}\right)^{2}+\left(x_{4}-(1-\beta) x_{2}\right)^{2}, \\
& A=\left(x_{2} y_{3}-x_{3} y_{2}\right)+\left(x_{4} y_{5}-x_{5} y_{4}\right)+K, \\
& \gamma=\frac{m_{\text {ura }}\left(1+m_{\text {sat }}\right)}{1+m_{\text {sat }}+m_{\text {ura }}} .
\end{aligned}
$$

\section{The computation of invariant tori}

In this section, we adapt a method for computing 2-D invariant tori (see Castellà \& Jorba 2000) to this situation. We are interested in finding a quasi-periodic solution (with two basic 
frequencies) of a given vector field. Note that this problem can be reduced to the one of finding an invariant curve of a suitable Poincaré map.

\subsection{Numerical computation of invariant curves}

Let be $\dot{x}=f(x)$ an autonomous vector field of dimension $n$, and $\Phi(x, t) \equiv \Phi_{t}(x)$ its associated flow. Let us define a Poincaré map as the time $T$-flow $\Phi_{T}(\cdot)$, where $T$ is a prefixed value. A continuous map $\varphi: \mathbb{T}^{1} \rightarrow \mathbb{R}^{n}$ is an invariant curve of the map $\Phi_{T}(\cdot)$ with rotation number $\omega$ iff

$\Phi_{T}(\varphi(\theta))-\varphi(\theta+\omega)=0$, for all $\theta \in \mathbb{T}^{1}$.

Note that we can expand $\varphi(\theta)$ as a real Fourier series,

$\varphi(\theta)=A_{0}+\sum_{k>0}\left(A_{k} \cos (k \theta)+B_{k} \sin (k \theta)\right), \quad A_{k}, B_{k} \in \mathbb{R}^{n}$.

Let us fix in advance a truncation value $N_{f}$ for this series (the selection of $N_{f}$ will be discussed in Sect. 3.2). To compute the $2 N_{f}+1$ unknown coefficients $A_{0}, A_{k}$ and $B_{k}, 1 \leq k \leq N_{f}$, we select an equally spaced mesh of $2 N_{f}+1$ points on $\mathbb{T}^{1}$,

$\theta_{j}=\frac{2 \pi j}{2 N_{f}+1} \quad 0 \leq j \leq 2 N_{f}$,

and, on this mesh, Eq. (6) reads,

$\Phi_{T}\left(\varphi\left(\theta_{j}\right)\right)-\varphi\left(\theta_{j}+\omega\right)=0, \quad 0 \leq j \leq 2 N_{f}$.

This is a nonlinear set of equations for the unknowns $A_{0}, A_{k}$ and $B_{k}$, that we will solve by means of a Newton method (the Jacobian of the map can be easily obtained from the chain rule).

Note that the number of equations is $\left(2 N_{f}+1\right) n$ and that the unknowns are $\left(A_{0}, A_{1}, B_{1}, \ldots, A_{N_{f}}, B_{N_{f}}\right), \omega$ and the time $T$ that defines the Poincaré map. Besides, note that if $\varphi(\theta)$ satisfies Eq. (6) then, for any $v, \varphi(\theta+v)$ also satisfies Eq. (6). Later we will discuss, for each case, how we deal with these degeneracies.

\subsection{Discretization error}

Once we have solved Eq. (8) with a certain tolerance (typically, $10^{-11}$ ), we still do not have any information on the error of the approximated invariant curve. This is because we have not estimated the discretization error coming from substituting Eqs. (6) by (8). A simple method to estimate the global error of the invariant curve is to estimate

$E(\varphi, \omega)=\max _{\theta \in \mathbb{T}}\left|\Phi_{T}(\varphi(\theta))-\varphi(\theta+\omega)\right|$

by using a mesh of points, say, 100 times finer than the mesh (7). If, for instance, $E(\varphi, \omega)>10^{-9}$, the solution obtained is not considered good enough and, therefore, the discretization order $N_{f}$ is increased and a new approximation is computed. The process is repeated until $E(\varphi, \omega)$ is lower than $10^{-9}$.

\section{The Bianular solution of the SJS system}

In this section, we will compute quasi-periodic solutions, with two basic frequencies, for the planar Sun-Jupiter-Saturn problem. Our main goal is to find an orbit such that the motion of Sun and Jupiter is as close as possible to the real one. In this case, as we are computing a 2-D torus, we have essentially two free parameters that will allow us to choose two osculating orbital elements of the relative Sun-Jupiter motion.

\subsection{The initial approximation}

In Gabern \& Jorba (2001), the authors computed a periodic solution, called the BCCP solution, for the planar Sun-JupiterSaturn Three-Body Problem. Here we use this solution as a basis to derive the initial approximation for a Newton method.

\subsubsection{The BCCP solution}

The BCCP solution is a periodic orbit of Hamiltonian (3) for the Sun-Jupiter-Saturn case, in which the two planets have the same mean motion as in the real system. The rotating Jacobi coordinates of a point of this orbit are shown in the following table,

\begin{tabular}{|l|l||l|l|}
\hline$q_{1}(0)$ & -0.999499455382433 & $p_{1}(0) / \alpha$ & 0.0 \\
$q_{2}(0)$ & 0.0 & $p_{2}(0) / \alpha$ & -1.00062422459750 \\
$q_{3}(0)$ & -1.83433334636804 & $p_{3}(0) / \beta$ & 0.0 \\
$q_{4}(0)$ & 0.0 & $p_{4}(0) / \beta$ & -0.738094038829119 \\
\hline
\end{tabular}

The linear normal behaviour of the orbit is given by the eigenvalues of the monodromy matrix,

\begin{tabular}{|r|r|r|r|}
\hline \multicolumn{1}{|c|}{$\operatorname{Re}(\lambda)$} & \multicolumn{1}{|c|}{$\pm \operatorname{Im}(\lambda)$} & $|\lambda|$ & $\pm \operatorname{Arg}(\lambda)$ \\
\hline \hline-0.4563371385719557 & 0.889806954322080 & 1.0 & 2.04467069171687 \\
\hline-0.4547032607925039 & 0.890642995046002 & 1.0 & 2.04283533844408 \\
\hline 1.0 & 0.0 & 1.0 & 0.0 \\
\hline 1.0 & 0.0 & 1.0 & 0.0 \\
\hline
\end{tabular}

As all of them have modulus one, the periodic orbit is linearly stable. Moreover, note that the matrix has two Jordan boxes corresponding to the angular momentum and energy integrals of motion. The use of the angular momentum to reduce one degree of freedom suppresses one of the Jordan boxes.

\subsubsection{On the two families of invariant curves}

It is known that, under generic conditions, each normal frequency of the periodic orbit gives rise to a Cantorian family of quasi-periodic motions with two basic frequencies, one coming from the frequency of the orbit and one from the normal mode considered. These families are usually called Lyapunov families (for more details see, for instance, Jorba \& Villanueva 1997, 2001).

For definiteness, we call Family1 the Lyapunov family corresponding to the eigenvalues $\left(\lambda_{1}, \bar{\lambda}_{1}\right)$ and Family 2 to the one corresponding to $\left(\lambda_{2}, \bar{\lambda}_{2}\right)$. In the next sections we will compute these two families, and we will look for solutions such that the 
motion of Jupiter has some prescribed characteristics. The concrete continuation procedure is explained in Sect. 4.3.

\subsubsection{Fixing some of the unknowns}

In what follows, $A_{k}$ and $B_{k}$ will denote the coefficients of the Fourier expansion of the invariant curve, and $A_{k}^{(l)}$ will refer to the component number $l$ of the vector $A_{k} \in \mathbb{R}^{6}$.

The prefixed unknowns at every step in the Newton method are, in this case,

$-T=T_{\text {sat }}=\frac{2 \pi}{\omega_{\text {at }}} ;$ we fix Saturn's frequency. With this selection of $T$ the points of the periodic orbit of the BCCP become fixed points of $\Phi_{T}$.

- $A_{0}^{(3)}=0$; we select one invariant curve on the torus.

$-A_{1}^{\left(l_{1}\right)}=\varepsilon\left(v_{1}^{\left(l_{1}\right)} \cos \left(\xi_{0}\right)+v_{2}^{\left(l_{1}\right)} \sin \left(\xi_{0}\right)\right) \neq 0$; we fix $\varepsilon$ to choose one of the quasi-periodic orbits of the Lyapunov family, and leave the rotation number $\omega$ to be self-adjusted.

- $A_{1}^{\left(l_{2}\right)}=\varepsilon\left(v_{1}^{\left(l_{2}\right)} \cos \left(\xi_{0}\right)+v_{2}^{\left(l_{2}\right)} \sin \left(\xi_{0}\right)\right)=0$; in order to have a unique parametrization for the curve. This condition can be satisfied choosing $\xi_{0} \in \mathbb{T}^{1}$ such that $\xi_{0}=\arctan \left(-v_{1}^{\left(l_{2}\right)} / v_{2}^{\left(l_{2}\right)}\right)$.

The last two conditions can be explained from the initial approximation that we choose in the Newton method. The indexes $l_{1}$ and $l_{2}$ depend on the family we are interested in. For instance, we have selected $l_{1}=3$ and $l_{2}=2$ for Family 1 and $l_{1}=2$ and $l_{2}=3$ for Family 2 .

Finally, the initial approximation for the Newton method is given by the linearization of the Poincare map $\Phi_{T}$ around the fixed point of $\Phi_{T}$ given in Sect. 4.1.1 (in what follows, we will refer to this point as $X_{0}$ ),

$$
\begin{array}{ll}
T=T_{\text {sat }}, & A_{1}=\varepsilon\left(\boldsymbol{v}_{1} \cos \left(\xi_{0}\right)+\boldsymbol{v}_{2} \sin \left(\xi_{0}\right)\right), \\
\omega=\lambda, & B_{1}=\varepsilon\left(\boldsymbol{v}_{1} \sin \left(\xi_{0}\right)+\boldsymbol{v}_{2} \cos \left(\xi_{0}\right)\right), \\
A_{0}=X_{0}, & A_{k}=0, k \geq 2, \\
& B_{k}=0, k \geq 2,
\end{array}
$$

where $\left(\lambda, v=\boldsymbol{v}_{\mathbf{1}}+\mathrm{i} \boldsymbol{v}_{2}\right)$ is one of the eigenvalue-eigenvector pairs of $D \Phi_{T}, \xi_{0}$ has been given before and $\varepsilon$ is a sufficiently small parameter (for instance, $\varepsilon \sim 10^{-3}$ ) chosen in order the invariant curve not to be too far from the periodic orbit.

\subsection{The first invariant torus for each family}

With the method described in Sect. 3 and the initial approximation given in the previous section, we have computed a first torus for each family (we call them Torus1 and Torus2, respectively). In Sect. 4.2.1 we show how to go back to the initial Jacobi coordinates and, in Sect. 4.2.2, we will discuss the orbital characteristics of these two initial orbits.

\subsubsection{Going back to rotating Jacobi coordinates}

In order to obtain the rotating Jacobi coordinates $\left(q_{j}, p_{j}\right)_{j=1 \div 4}$ from the reduced variables $\left(x_{j}, y_{j}\right)_{j=1 \div 3}$ and the angular momentum $K$ in a unique way, we have to select an extra condition. Note that, in the reduction process, the angle between the Sun-Jupiter vector and the $x$-axis is eliminated. Thus, if we want to invert this change we have to decide how to fix the relative position of the $x$-axis.

From now on, when we undo the reduction change, we fix the $x$-axis in the following way: we find the angle $\theta$ for which $x_{3}(\theta)=0$ (that is, when Sun, Jupiter and Saturn are in a particular collinear configuration) and we set the rotating Jacobi coordinates as:

$$
\begin{array}{|l|l|}
\hline q_{1}=-x_{1} & p_{1}=-\alpha x_{4} \\
q_{2}=0 & p_{2}=-\frac{A}{x_{1}} \\
q_{3}=x_{2} & p_{3}=\beta x_{5} \\
q_{4}=-x_{3} & p_{4}=-\beta x_{6} \\
\hline
\end{array}
$$

where $x_{4}, x_{5}$ and $x_{6}$ are such that $y_{1}=\alpha x_{4}, y_{2}=\beta x_{5}$ and $y_{3}=\beta x_{6}$. Recall that $A=\beta\left(x_{2} x_{6}-x_{3} x_{5}\right)+K, \alpha=\mu(1-\mu)$ and $\beta=\frac{m_{\text {sat }}}{1+m_{\text {sat }}}$.

\subsubsection{Some osculating orbital elements}

Here we take a point on each torus and we apply the previous transformation to send them to the rotating Jacobi coordinates. We use the resulting initial condition for a numerical integration of the Hamiltonian (3) with $N=2$ during a time span of 50 Jupiter sidereal revolutions. The concrete selection of initial condition on the torus does not make a big difference in the calculations.

The projection for Torus1 and Torus 2 into the $\left(q_{1}, q_{2}\right)$, $\left(p_{1}, p_{2}\right)$ and $\left(q_{3}, q_{4}\right)$ planes are shown in Fig. 1 . We can see (left and center plots) that the relative Sun-Jupiter motion is a libration around the point $\left(q_{1}, q_{2}, p_{1}, p_{2}\right)=(-1,0,0,-1)$.

The evolution of Jupiter's and Saturn's osculating semimajor axis, eccentricity and perihelion argument for Torus 1 and Torus2 are displayed in Figs. 2 and 3. The integration time, in this case, is 5 periods of Saturn.

Note that the semi-major axis of Jupiter's and Saturn's orbits in either Torus 1 or Torus 2 are quite well adjusted (our unit of length is the Sun-Jupiter distance) and, in consequence, their periods (that oscillate around $2 \pi$ and $\frac{2 \pi}{\omega_{\text {sat }}}$, respectively) are also closed to the actual ones. This was expected because, during the computations, the time $T$ of the Poincaré map has been fixed to the period of Saturn.

The osculating eccentricities of Jupiter and Saturn are too small $\left(10^{-3}\right.$, approximately) compared to the actual ones. Thus, the next step is to find a torus, either in Family1 or Family2, for which the eccentricity of Jupiter is closer to the actual one.

\subsection{Continuation of the two families of invariant tori}

As it is well known, the families of invariant tori are not continuous but Cantorian. However, it is quite common in many situations that the holes that conform the Cantor structure are too small to be detected by the standard double precision arithmetic of the computer (see Jorba \& Villanueva (1997) for some analytical estimates, and Broer et al. (2003) for a discussion on the effect of the different kind of resonances). Therefore, except by low order resonances, these families look continuous in the computer. 

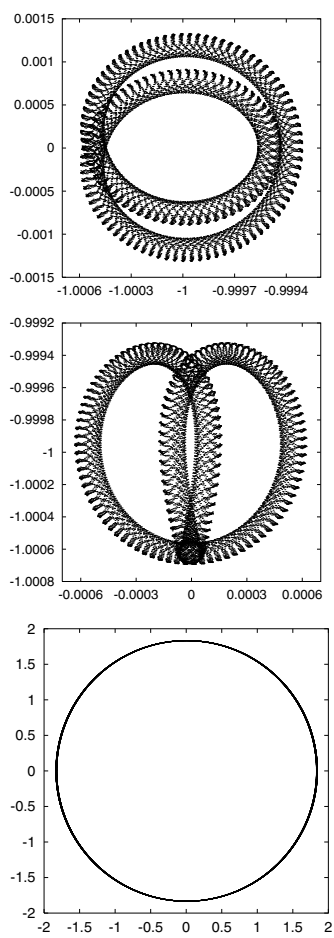

Fig. 1. Top: Sun-Jupiter relative motion for Torus1 (left) and Torus2 $($ right). Center: momenta for the Sun-Jupiter relative motion for Torus1 (left) and Torus2 (right). Bottom: Saturn's orbit around the Sun-Jupiter barycenter for Torus1 (left) and Torus2 (right).

Once we have a first torus, we want to continue the family it belongs to. This family can be parameterized by the angular momentum $K$. It is easy to see (and also checked from the computations) that there is a strong relationship between the angular momentum, $K$, and the osculating orbital elements of Jupiter's and Saturn's orbits.

As we want to simulate in a more realistic way the SunJupiter relative motion, we are more interested in adjusting Jupiter's orbital elements than Saturn's ones (although it is possible to adjust Saturn's instead). As there is one free parameter (we are allowed to set $K$ ), we select the osculating eccentricity of Jupiter's orbit as the target value. Thus, by means of a continuation method, we try to find another torus inside Family 1 or Family 2 for which the osculating eccentricity of Jupiter is approximately 0.0484 .

\subsubsection{Method of continuation}

The continuation process starts with the Torus 1 or Torus 2 computed in Sect. 4.2, depending on the family that we want to follow (Family1 or Family2, respectively).

To compute a torus belonging to a particular family, we use a small modification of the method explained in Sect. 3. We add to Eq. (8) a new one:

$\operatorname{eccen}\left(x_{1}, x_{2}, x_{3}, x_{4}, x_{5}, x_{6}, K\right)=$

$$
\sqrt{1+\frac{A^{4}}{\alpha^{4} x_{1}^{2}}+\frac{A^{2} x_{4}^{2}}{\alpha^{2}}-2 \frac{A^{2}}{\alpha^{2} x_{1}}}=e,
$$


Fig. 2. From top to bottom: evolution in Torus1 of the osculating semimajor axis, eccentricity and argument of the perihelion of Jupiter's orbit (left) and Saturn's orbit (right) in a time span of 5 Saturn's sidereal revolutions.
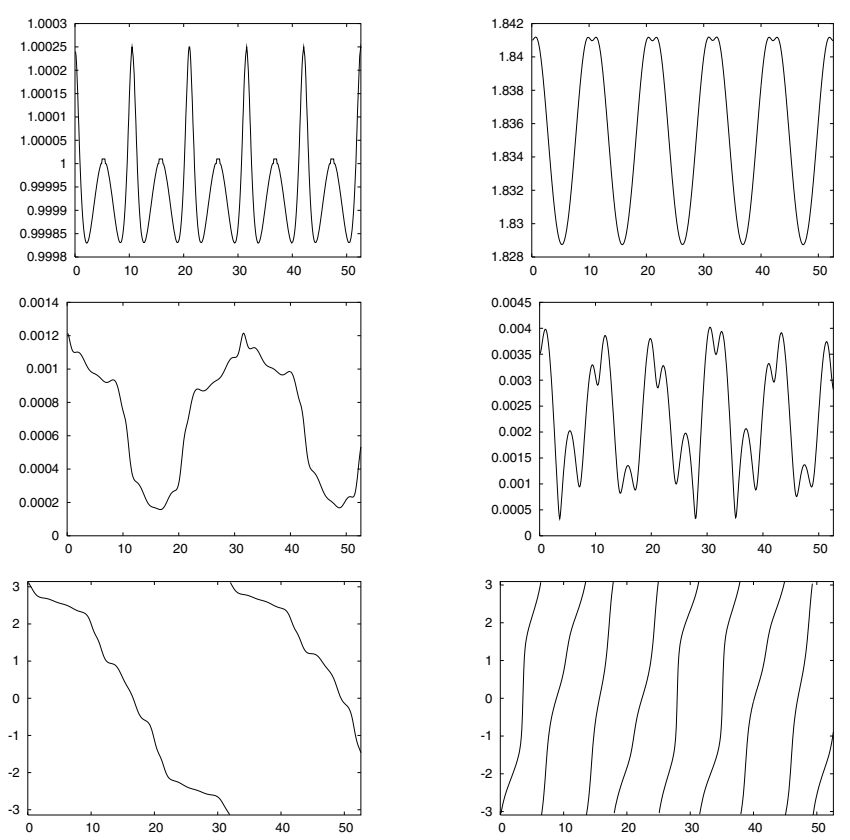

Fig. 3. From top to bottom: evolution in Torus 2 of the osculating semimajor axis, eccentricity and argument of the perihelion of Jupiter's orbit (left) and Saturn's orbit (right) in a time span of 5 Saturn's sidereal revolutions.

where $\alpha=\mu(1-\mu), A=\beta\left(x_{2} x_{6}-x_{3} x_{5}\right)+K$, eccen $(\cdot)$ is a function that gives us Jupiter's osculating eccentricity from the reduced coordinates in a given moment (we evaluate it when Sun, Jupiter and Saturn are in a particular collinear configuration; more concretely, when $x_{3}=0$ ) and $e$ is a fixed constant that will be used as a continuation parameter. 

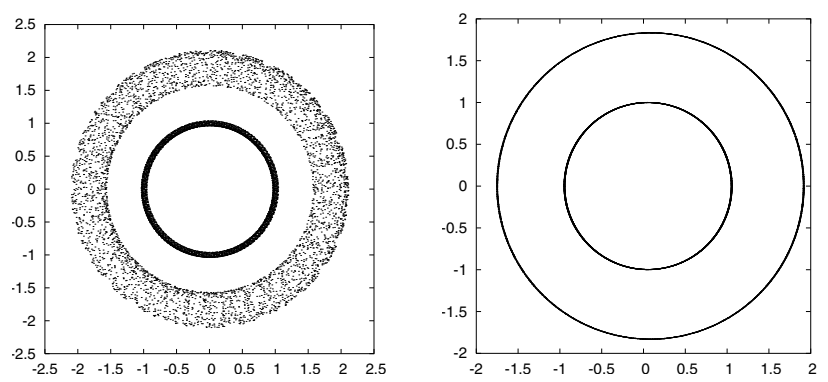

Fig. 4. Left: projection into the configuration space of the torus belonging to Family1 when the continuation procedure is stopped. Right: projection into the configuration inertial space of the final torus of Family2. See the text for more details.

Thus, at each step of the continuation scheme, we find an invariant curve of the Poincaré map $\Phi_{T}(\cdot)$, a truncation number of the Fourier series $\left(N_{f}\right)$, a rotation number $(\omega)$ and an angular momentum $(K)$ that are solution of Eqs. (8) and (9) for a fixed eccentricity $(e)$. As explained in Sect. 3.1, every invariant curve is refined by means of a Newton method, and in each step of the Newton method we solve a non-squared linear system using a least-squares method.

During the continuation process, the parameters that we keep constant are slightly different from those in Sect. 4.1.3:

- $T=T_{\text {sat }}=\frac{2 \pi}{\omega_{\text {sat }}} ;$ we fix Saturn's frequency.

$-A_{0}^{(3)}=0$; we fix the invariant curve on the torus.

- $\omega$; we fix the rotation number.

- $A_{1}^{\left(l_{2}\right)}=0$; in order to have a unique parametrization for the curve.

- $e$; we fix the eccentricity, we do not impose any condition on the angular momentum $K$.

where $l_{2}=2$ for Family 1 and $l_{2}=3$ for Family 2 .

We stress that the continuation parameter is $e$ and we want to increase it from the value that it initially has for Torus 1 and Torus2 $\left(10^{-3}\right.$ approximately) to the value $e=0.0484$.

\subsubsection{Results: The Bianular solution}

First, we start from Torus 1 and we continue Family 1 by increasing the parameter $e$. We see that, when $e$ increases, the number of harmonics $\left(N_{f}\right)$ has to be increased if we want the discretization error of the invariant curve to be smaller than a certain tolerance (typically, we take $10^{-9}$ ). We stop the continuation process when $N_{f}$ reaches 90 . At this moment, the osculating eccentricity of Jupiter's orbit is approximately 0.036 and if we look at the other orbital elements of Jupiter's and Saturn's orbits, we see that they do not evolve in the desired direction, but they move away from the real ones. In Fig. 4 (left), the projection of this solution into the configuration space is shown. This orbit is far from a planetary one because, for example, the big variation of the two semi-major axis. Thus, increasing Jupiter's eccentricity inside Family1 forces us to move away from the desired solution. In consequence, it is not possible to find an adequate torus in this family.

For Family2, we proceed in the same way as before but starting from Torus2. In this case, we are able to increase $e$ up

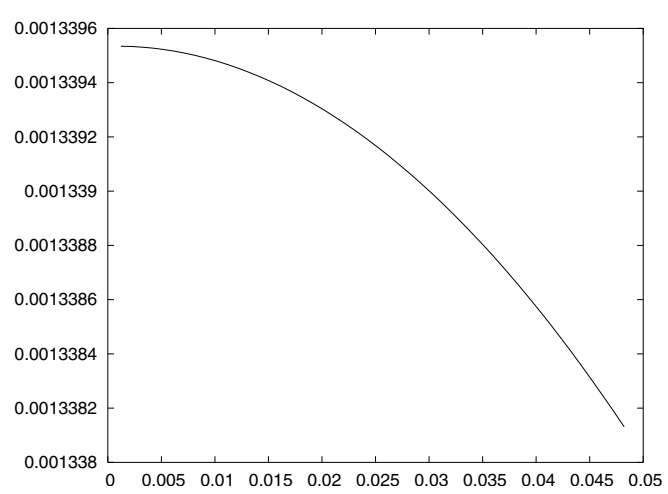

Fig. 5. Plot of the evolution of the angular momentum $K$ (vertical axis) when the parameter $e$ (horizontal axis) is increased from 0.00121 (corresponding to Torus2) to 0.0484 (the desired value) in the continuation of Family2.
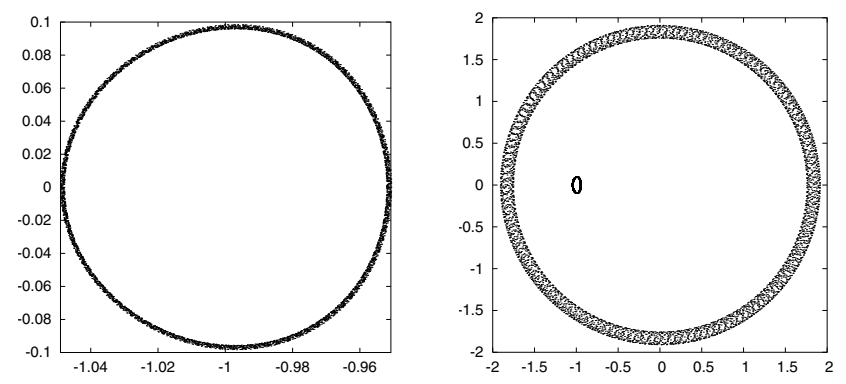

Fig. 6. Left: $\left(x_{1}, x_{2}\right)$-projection of the Sun-Jupiter relative orbit on the torus. Right: Saturn's orbit around the Sun-Jupiter barycenter. Inside it, we can see the Sun-Jupiter relative orbit. This is what we call "Bianular solution" of the Sun-Jupiter-Saturn planar Three Body Problem.

to the value 0.0484 , because the number of harmonics does not grow up very much (actually, if we ask the invariant curve to have an error smaller than $10^{-9}, N_{f}$ increases from 6 to 9) and the solution obtained is of the planetary type (see Fig. 4, right). In Fig. 5, we plot the variation of the angular momentum $K$ of the planar SJS Three Body Problem when the parameter $e$ is increased in the continuation process.

We can see the projection of the final torus on the rotating Jacobi configuration coordinates $\left(q_{1}, q_{2}, q_{3}, q_{4}\right)$ in Fig. 6. This solution of the planar Sun-Jupiter-Saturn TBP is what we call the Bianular solution of the TBP. This torus is parameterized with the angles $\left(\theta_{1}, \theta_{2}\right)=\left(\omega_{1} t+\theta_{1}^{0}, \omega_{2} t+\theta_{2}^{0}\right)$, where the frequencies are $\omega_{1}=\omega_{\text {sat }}=0.597039074021947$ and $\omega_{2}=\frac{\omega_{1} \bar{\omega}}{2 \pi}=0.194113943490717$, and $\theta_{1,2}^{0}$ are the initial phases.

We have also plotted the evolution of the osculating orbital elements of Jupiter and Saturn in a time span of $5 T_{\text {sat }}$ adimensional units in Fig. 7.

Recall that our main goal was to simulate in a realistic way the relative Sun-Jupiter motion. We have obtained (numerically) a quasi-periodic solution of the TBP where the osculating eccentricity, the semi-major axis, the period and the mean motion of Jupiter's orbit are quite well adjusted.

Concerning Saturn's motion on the torus, we have quite well fitted the semi-major axis and the period of its orbit; the 

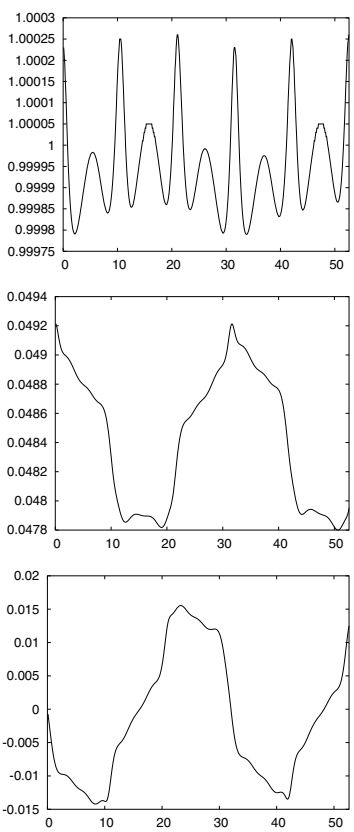
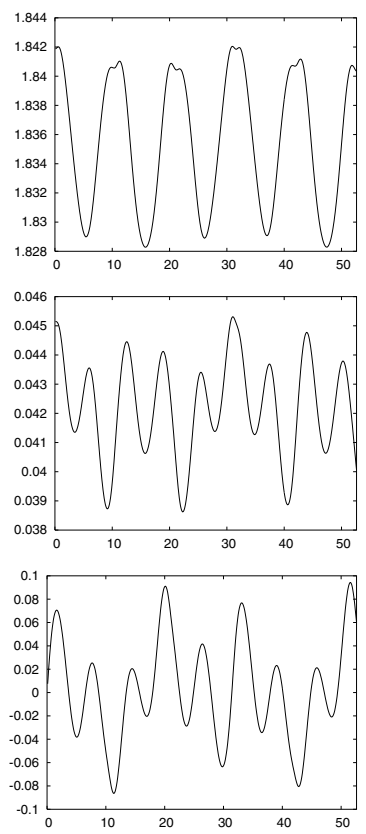

Fig. 7. From top to bottom: evolution of the osculating semi-major axis, eccentricity and argument of the perihelion of Jupiter's orbit (left) and Saturn's orbit (right) in a time span of 5 Saturn revolutions around the Sun.

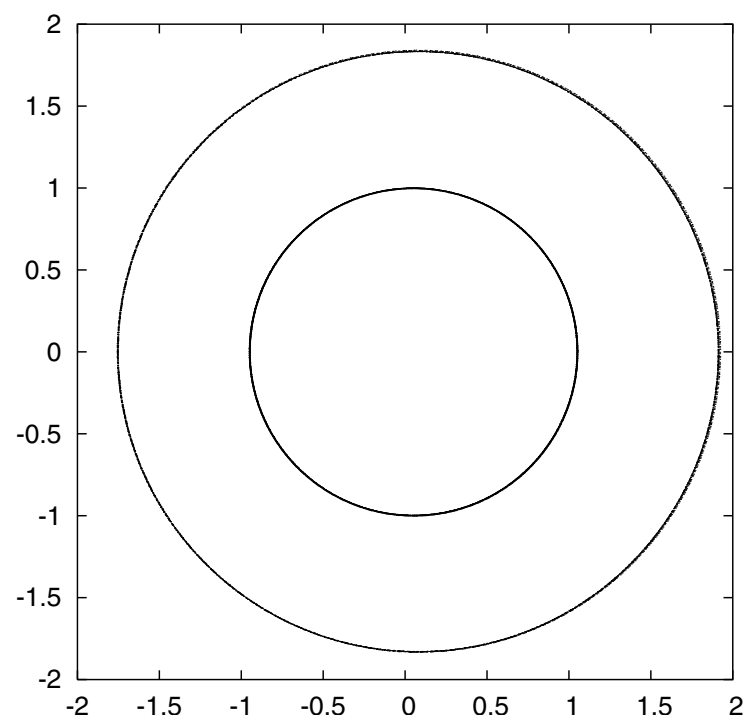

Fig. 8. Superposition, in the configuration space, of the real motion of Jupiter and Saturn and the Bianular solution. The two orbits are too similar to see any difference at this scale. See the text for more details.

obtained eccentricity is about $80 \%$ of the actual value and the argument of the perihelion still oscillates too much.

In Fig. 8, we compare the result of a numerical integration of the Sun-Jupiter-Saturn system (using the real initial conditions for the three bodies) with the Bianular solution. The agreement is quite good.

An alternative approach is to compute a resonant solution of the TBP (see Hadjidemetriou 1980). In this case, the frequencies are not the actual ones but very close to them; for instance, if we consider the 5:2 resonance between the orbits of Jupiter and Saturn, we should take $\omega_{\text {sat }}^{\prime}=1-\frac{2}{5}=0.6$ instead of the actual value $\omega_{\text {sat }}=0.597039074$.

\section{A quasi-periodic solution of the SJSU problem}

In this section, we compute a quasi-periodic solution with 2 frequencies of the planar SJSU four body problem given by the Hamiltonian (5). We will adapt the method described in Sect. 3 for computing invariant curves of maps to this case.

First, in Sect. 5.1, we will give an heuristic approximation of the initial point used in the Newton method for computing an invariant curve when $m_{\text {ura }}=0 . m_{\text {ura }}=0$ means that Uranus does not affect the motion of Sun, Jupiter and Saturn, but it is affected by them. Then, in Sect. 5.2 we will compute a first torus for $m_{\text {ura }}=0$. In Sect. 5.3, by means of a continuation method (taking $m_{\text {ura }}$ as the parameter) we compute a quasi-periodic solution with 2 frequencies of the SJSU planar problem.

\subsection{First approximation}

As a first approximation, we suppose that Uranus has zero mass and that it is moving in a Keplerian orbit around the Sun. If we set that, at $t=0$, Sun, Jupiter, Saturn and Uranus are in a particular collinear configuration (for instance, on the line $y=0$ ), it is easy to obtain an approximate initial condition for Uranus in the rotating Jacobi coordinates as a Keplerian solution of the two body problem formed by the center of masses of the system and Uranus. The initial conditions for Uranus are then

\begin{tabular}{|l|l||l|l|}
\hline$q_{5}$ & -3.6799515105016547 & $p_{5} / \gamma$ & 0.0 \\
$q_{6}$ & 0.0 & $p_{6} / \gamma$ & -0.521289469537814 \\
\hline
\end{tabular}

On the other hand, we take the periodic orbit of the planar Sun-Jupiter-Saturn TBP computed in Gabern \& Jorba (2001) (that has already been used in Sect. 4.1.1 as the initial approximation for the Bianular solution). The period of this orbit is $T=T_{\text {sat }}=\frac{2 \pi}{\omega_{\text {sat }}}$, where $\omega_{\text {sat }}=0.597039074021947$ is the relative frequency of Saturn in the Sun-Jupiter rotating system. As in Sect. 4.1.1, we take the coordinates of the point of this orbit for which the three bodies are in the chosen collinear configuration:

\begin{tabular}{|l|l||l|l|}
\hline$q_{1}$ & -0.999499455382433 & $p_{1} / \alpha$ & 0.0 \\
$q_{2}$ & 0.0 & $p_{2} / \alpha$ & -1.00062422459750 \\
$q_{3}$ & -1.83433334636804 & $p_{3} / \beta$ & 0.0 \\
$q_{4}$ & 0.0 & $p_{4} / \beta$ & -0.738094038829119 \\
\hline
\end{tabular}

If we integrate the flow corresponding to Hamiltonian (3), with $N=3$, in the time interval $t \in\left[0, T_{\text {sat }}\right]$ taking as initial condition the point of the periodic orbit for the Sun-Jupiter-Saturn system and the Keplerian approximation for Uranus, we find 
that the orbit corresponding to Sun, Jupiter and Saturn obviously closes (it is a periodic orbit of period $T_{\text {sat }}$ ) and the one corresponding to Uranus does not close and turns one lap-odd.

We are interested in measuring the angle swept by Uranus during $T_{\text {sat }}$ units of time. Note that this angle is very close to the following number:

$\tilde{\omega}=\frac{2 \pi \omega_{\text {ura }}}{\omega_{\text {sat }}} \bmod 2 \pi=2.750807556$,

where $\omega_{\text {ura }}=0.858425538978989$ is the relative frequency of Uranus in the Sun-Jupiter rotating system. Thus, note that, if we keep constant the value $T_{\text {sat }}$ and if we impose $\tilde{\omega}$ to be the rotation number of the invariant curve, the two frequencies of the 2 -D invariant torus that we are computing will be $\omega_{\text {sat }}$ (Saturn's frequency) and $\omega_{\text {ura }}$ (Uranus's frequency).

\subsection{A torus in the SJSU system with a massless Uranus}

Here, using the method described in Sect. 3, we will compute a 2-D invariant torus for the reduced planar SJSU problem (see Eq. (5)) in the case $m_{\text {ura }}=0$.

\subsubsection{Solving the invariant curve equations}

As before, $A_{k}$ and $B_{k}$ will denote the coefficients of the Fourier expansion of the invariant curve, and $A_{k}^{(l)}$ will refer to the component number $l$ of the vector $A_{k} \in \mathbb{R}^{6}$.

To solve Eq. (8) we use a Newton method. As in the SJS case the system is degenerated, so we will fix some of the unknowns:

- $T=T_{\text {sat }}$; we fix Saturn's frequency.

$-\omega=\tilde{\omega}$; to select the rotation number (this implies that the frequency of Uranus is fixed).

- $A_{0}^{(3)}=0$; to select one invariant curve on the torus.

$-A_{1}^{(5)}=0$; to have a unique parametrization for the curve.

\subsubsection{First approximation}

As initial approximation of the Newton method for solving Eq. (8) in the case $m_{\text {ura }}=0$, we use the orbit described in Sect. 5.1. To do that, we write the initial point of this orbit in the reduced coordinates of the 3-Planetary problem (5),

\begin{tabular}{|l|l||r|l|}
\hline$x_{1}$ & 0.999499455382433 & $x_{6}$ & 0.0 \\
$x_{2}$ & -1.83433334636804 & $x_{7}$ & 0.0 \\
$x_{3}$ & 0.0 & $x_{8}$ & 0.7380940388291189 \\
$x_{4}$ & -3.679951510501655 & $x_{9}$ & 0.0 \\
$x_{5}$ & 0.0 & $x_{10}$ & 0.5212894695378142 \\
\hline
\end{tabular}

where the new coordinates $x_{6}, \ldots, x_{10}$ are now defined such that $y_{1}=\alpha x_{6}, y_{2}=\beta x_{7}, y_{3}=\beta x_{8}, y_{4}=\gamma x_{9}$ and $y_{5}=\gamma x_{10}$.
If we denote this point as $X_{0}\left(X_{0}=\left(x_{1}, \ldots, x_{10}\right)\right)$, we have already shown in Sect. 5.1 that

$$
\Phi_{T}\left(X_{0}\right) \approx\left(\begin{array}{c}
x_{1} \\
x_{2} \\
x_{3} \\
\cos \left(\omega_{0}\right) x_{4}-\sin \left(\omega_{0}\right) x_{5} \\
\sin \left(\omega_{0}\right) x_{4}+\cos \left(\omega_{0}\right) x_{5} \\
x_{6} \\
x_{7} \\
x_{8} \\
\cos \left(\omega_{0}\right) x_{9}-\sin \left(\omega_{0}\right) x_{10} \\
\sin \left(\omega_{0}\right) x_{9}+\cos \left(\omega_{0}\right) x_{10}
\end{array}\right) .
$$

Thus, taking as initial approximation of the invariant curve for the first step in the Newton process the values

$A_{0}=\left(\begin{array}{c}x_{1} \\ x_{2} \\ x_{3} \\ 0 \\ 0 \\ x_{6} \\ x_{7} \\ x_{8} \\ 0 \\ 0\end{array}\right), \quad A_{1}=\left(\begin{array}{c}0 \\ 0 \\ 0 \\ x_{4} \\ x_{5} \\ 0 \\ 0 \\ 0 \\ x_{9} \\ x_{10}\end{array}\right), \quad B_{1}=\left(\begin{array}{c}0 \\ 0 \\ 0 \\ -x_{5} \\ x_{4} \\ 0 \\ 0 \\ 0 \\ -x_{10} \\ x_{9}\end{array}\right)$,

$A_{k}=B_{k}=0, k \geq 2$, and $\omega=\tilde{\omega}$, the method converges to an invariant curve of the Poincaré map $\Phi_{T_{\text {sat }}}$ of the vector field (5) with $m_{\text {ura }}=0$. The two frequencies of this quasi-periodic orbit are $\omega_{1}=\omega_{\text {sat }}$ and $\omega_{2}=\omega_{\text {ura }}$.

\subsection{The Tricircular solution of the SJSU problem}

Once we have computed an invariant torus for the case $m_{\text {ura }}=0$, we proceed by a continuation method to increase the parameter $m_{\text {ura }}$ up to its actual value. During the continuation, the two frequencies, $\omega_{1}=\omega_{\text {sat }}$ and $\omega_{2}=\omega_{\text {ura }}$ are kept constant. Thus, we obtain a quasi-periodic solution (that moves on a 2-D torus parameterized by the two angles $\theta_{1}=\omega_{1} t+\theta_{1}^{0}$ and $\theta_{2}=\omega_{2} t+\theta_{2}^{0}$ ) of the reduced four body field (5).

To invert the reduction change we will proceed as in Sect. 4.2.1. Note that during the reduction of one degree of freedom by using the angular momentum the angle between the $P_{0} P_{1}$ direction and the $x$-axis has been lost. Therefore, we have imposed that the $x$-axis is such that at $t=0$, the Sun, Jupiter and Saturn are in a particular collinear configuration (actually, on the line $y=0$ ).

In Fig. 9, we have plotted the projection into the configuration space of this torus in a rotating (left plot) and in an inertial (right plot) frame. Note that the shape of this plot is very close to three circles. This is the reason for calling this solution Tricircular solution of the SJSU four body problem.

\section{The Hamiltonians of the BAP and TCCP models}

In this section, we generalize the procedure used in Gabern \& Jorba (2001) for constructing the BCCP model as a (timedependent) perturbation of the RTBP from a particular solution 

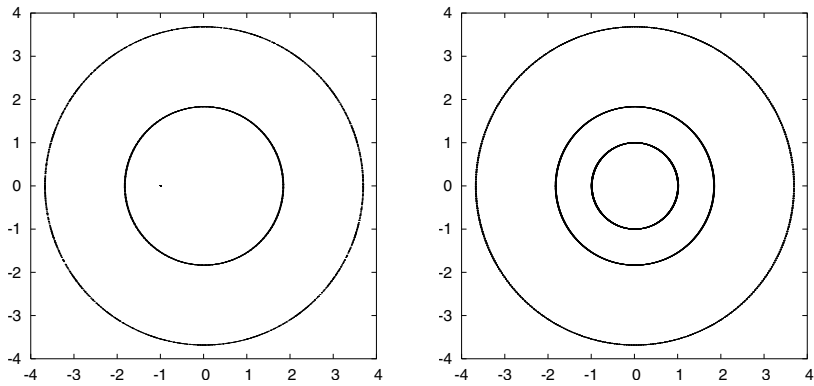

Fig. 9. Quasi-periodic solution for the Four Body Sun-Jupiter-SaturnUranus Problem. The exterior orbit corresponds to Uranus, the one in the middle to Saturn and the interior one (seen as a small point that librates around the point $(-1,0)$, in the left plot) is the relative SunJupiter's orbit. The left plot corresponds to the rotating coordinates and the right one is represented in an inertial reference frame.

of the three body problem. These ideas have already been used in Gómez et al. (2001a,b,c,d) and also in Gómez et al. (2002).

Thus, we construct the Hamiltonian of a massless particle that moves under the attraction of the main $N+1$ bodies in any solution of the $N$-planetary problem (1) and we write it as a quasi-periodic time-dependent perturbation of the RTBP, with Sun and Jupiter as primaries.

Afterwards, we compute the coefficients of the quasiperiodic functions appearing in the Hamiltonian for the Bianular and Tricircular solutions of the two and three planetary problems.

\subsection{Hamiltonian of the restricted $\mathrm{N}$-planetary problem}

Let us suppose that we know a solution of the planar $N$-planetary problem (1), and let $\left(\boldsymbol{q}_{1}, \ldots, \boldsymbol{q}_{N}, \boldsymbol{p}_{1}, \ldots, \boldsymbol{p}_{N}\right)$ be its representation in an inertial three dimensional reference frame (where the vertical components of $\boldsymbol{q}_{i}$ and $\boldsymbol{p}_{i}$ have been set to zero). Let $\boldsymbol{q}_{\text {part }}$ be the position vector of the particle in this inertial reference frame.

We will use the the following three transformations.

a) Translation of the origin from the global barycentre to the Sun-Jupiter barycentre;

b) rotation to fix Sun and Jupiter on the $x$-axis;

c) time-dependent scaling to force the distance between Sun and Jupiter to be one.

In this "rotating-pulsating" reference frame, the main bodies and the particle have the coordinates:

Sun: $\boldsymbol{Q}_{0}=\left(\delta_{1}, 0,0\right)^{t}$,

Jupiter: $\boldsymbol{Q}_{1}=\left(\delta_{1}-1,0,0\right)^{t}$,

Planets: $\boldsymbol{Q}_{k}=\frac{1}{\left\|\boldsymbol{q}_{1}\right\|} C^{t}\left(\boldsymbol{q}_{k}+\sum_{i=2}^{k-1} \delta_{i} \boldsymbol{q}_{i}\right), \quad k=2, \ldots, N$,

particle: $\boldsymbol{r}=\frac{1}{\left\|\boldsymbol{q}_{1}\right\|} C^{t}\left(\boldsymbol{q}_{\mathrm{part}}+\sum_{i=2}^{N} \delta_{i} \boldsymbol{q}_{i}\right)$, where $\delta_{i}=\frac{m_{i}}{\eta_{i}}$ and we recall that $\eta_{i}=\sum_{j=0}^{i} m_{j}$. The matrix $C$ is defined as

$C=\left(\begin{array}{ccc}-\frac{q_{1}^{(x)}}{\left\|\boldsymbol{q}_{1}\right\|} & \frac{q_{1}^{(y)}}{\left\|\boldsymbol{q}_{1}\right\|} & 0 \\ -\frac{q_{1}^{(y)}}{\left\|\boldsymbol{q}_{1}\right\|} & -\frac{q_{1}^{(x)}}{\left\|\boldsymbol{q}_{1}\right\|} & 0 \\ 0 & 0 & 1\end{array}\right)$

where $\boldsymbol{q}_{i}=\left(q_{i}^{(x)}, q_{i}^{(y)}, 0\right)_{i=1, \ldots, N}$ is the solution of the $N$-planetary problem embedded in the three dimensional configuration space. The inverse change is given by

$\boldsymbol{q}_{\mathrm{part}}=B \boldsymbol{r}-\sum_{i=2}^{N} \delta_{i} \boldsymbol{q}_{i}$,

where $B=\left\|\boldsymbol{q}_{1}\right\| C$. Defining the conjugate momentum as

$\boldsymbol{p}=\left\|\boldsymbol{q}_{1}\right\|^{2} \dot{\boldsymbol{r}}+B^{t} \dot{B} \boldsymbol{r}$,

the equations for the particle become Hamiltonian, and the Hamiltonian is:

$H=\frac{1}{2\left\|\boldsymbol{q}_{1}\right\|^{2}}\langle\boldsymbol{p}, \boldsymbol{p}\rangle-\frac{1}{\left\|\boldsymbol{q}_{1}\right\|^{2}} \boldsymbol{p} B^{t} \dot{B} \boldsymbol{r}-\sum_{i=2}^{N} \delta_{i} \ddot{\boldsymbol{q}}_{i}{ }^{t} B \boldsymbol{r}+U$,

where $U$, the potential energy, is given by

$U=-\sum_{i=0}^{N} G \frac{m_{i}}{\left\|\boldsymbol{q}_{1}\right\|} \frac{1}{\left\|\boldsymbol{Q}_{i}-\boldsymbol{r}\right\|}$

To write the Hamiltonian in a more suitable way, we look at the $(x, y)$ plane as if it were the complex plane, so that we write the vectors $\boldsymbol{q}_{i}$ as $z_{i}=q_{i}^{(x)}+i q_{1}^{(y)}$, for $i=1, \ldots, N$. Now we define the functions

$\alpha_{1}=\frac{1}{\left\|\boldsymbol{q}_{1}\right\|^{2}}$

$\alpha_{2}=\frac{-\operatorname{Re}\left(\dot{z}_{1} \bar{z}_{1}\right)}{\left\|\boldsymbol{q}_{1}\right\|^{2}}$

$\alpha_{3}=\frac{\operatorname{Im}\left(\dot{z}_{1} \bar{z}_{1}\right)}{\left\|\boldsymbol{q}_{1}\right\|^{2}}$,

$\alpha_{4}=\sum_{i=2}^{N} \delta_{i} \operatorname{Re}\left(\ddot{z}_{i} \bar{z}_{1}\right)$,

$\alpha_{5}=\sum_{i=2}^{N} \delta_{i} \operatorname{Im}\left(\ddot{z}_{i} \bar{z}_{1}\right)$

$\alpha_{6}=\frac{1}{\left\|\boldsymbol{q}_{1}\right\|}$,

$\alpha_{3+2 k}=\frac{-1}{\left\|\boldsymbol{q}_{1}\right\|^{2}} \operatorname{Re}\left(z_{k} \bar{z}_{1}+\sum_{i=2}^{k-1} \delta_{i} z_{i} \bar{z}_{1}\right)$,

$\alpha_{4+2 k}=\frac{-1}{\left\|\boldsymbol{q}_{1}\right\|^{2}} \operatorname{Im}\left(z_{k} \bar{z}_{1}+\sum_{i=2}^{k-1} \delta_{i} z_{i} \bar{z}_{1}\right)$,

where $k=2, \ldots, N$. Note that the functions $\alpha_{i}$ depend on time in a quasi-periodic way, with the same set of basic frequencies $\varpi=\left(\omega_{1}, \ldots, \omega_{m}\right)$ as the solution $\left(\boldsymbol{q}_{i}\right)_{i=1 \div N}$ of the $N$-planetary problem. It is then possible to write the Hamiltonian of the restricted $N$-planetary problem as:

$$
\begin{aligned}
H= & \left\langle\varpi, p_{\theta}\right\rangle+\frac{1}{2} \alpha_{1}(\theta)\left(p_{x}^{2}+p_{y}^{2}+p_{z}^{2}\right) \\
& +\alpha_{2}(\theta)\left(x p_{x}+y p_{y}+z p_{z}\right)+\alpha_{3}(\theta)\left(y p_{x}-x p_{y}\right) \\
& +\alpha_{4}(\theta) x+\alpha_{5}(\theta) y-\alpha_{6}(\theta) \sum_{i=0}^{N} G \frac{m_{i}}{\rho_{i}}
\end{aligned}
$$

where $\rho_{0}^{2}=\left(x-\delta_{1}\right)^{2}+y^{2}+z^{2}, \rho_{1}^{2}=\left(x-\delta_{1}+1\right)^{2}+y^{2}+z^{2}, \rho_{k}^{2}=$ $\left(x-\alpha_{3+2 k}(\theta)\right)^{2}+\left(y-\alpha_{4+2 k}(\theta)\right)^{2}+z^{2}$ for $k=2, \ldots, N$, and $p_{\theta}=$ $\left(p_{\theta_{1}}, \ldots, p_{\theta_{m}}\right)$ are the conjugate momenta of $\theta=\left(\theta_{1}, \ldots, \theta_{m}\right)$. 


\subsection{The Bianular problem}

Let

$\varphi(\theta)=A_{0}+\sum_{j=1}^{N_{f}}\left(A_{j} \cos j \theta+B_{j} \sin j \theta\right)$

be a parameterization of the invariant curve computed in Sect. 4. Note that, if we take the points on this curve as initial conditions for a numerical integration of the flow (4), the corresponding orbits will come back to this curve after $T_{\text {sat }}$ units of time. Therefore, a parameterization $X\left(\theta_{1}, \theta_{2}\right)$ of this 2-D torus can be computed from the following relation,

$X\left(\theta_{1}, \theta_{2}\right)=\Phi_{\frac{\theta_{1}}{\omega_{1}}}\left(\varphi\left(\theta_{2}-\frac{\omega \theta_{1}}{2 \pi}\right)\right)$,

where $\omega_{1}=\omega_{\text {sat }}$. It is very easy to check that $X\left(\theta_{1}, \theta_{2}\right)$ is $2 \pi$-periodic in each of its arguments:

$$
\begin{aligned}
X\left(\theta_{1}+2 \pi, \theta_{2}+2 \pi\right) & =\Phi_{\frac{\theta_{1}+2 \pi}{\omega_{1}}}\left(\varphi\left(\theta_{2}+2 \pi-\frac{\omega \theta_{1}}{2 \pi}-\omega\right)\right) \\
& =\Phi_{\frac{\theta_{1}}{\omega_{1}}}\left(\Phi_{T_{\text {sat }}}\left(\varphi\left(\theta_{2}-\frac{\omega \theta_{1}}{2 \pi}-\omega\right)\right)\right) \\
& =\Phi_{\frac{\theta_{1}}{\omega_{1}}}\left(\varphi\left(\theta_{2}-\frac{\omega \theta_{1}}{2 \pi}\right)\right)=X\left(\theta_{1}, \theta_{2}\right) .
\end{aligned}
$$

Therefore, it is trivial to tabulate $X\left(\theta_{1}, \theta_{2}\right)$ on a regular mesh

$\left(\theta_{1}^{j_{1}}, \theta_{2}^{j_{2}}\right)_{j_{1}, j_{2}=1 \div N_{t}}=\left(\frac{2 \pi j_{1}}{N_{t}}, \frac{2 \pi j_{2}}{N_{t}}\right)$.

On the other hand, it is also a straightforward computation to write the functions $\alpha_{i}$ that appear in the Hamiltonian (11) in terms of the solution $X\left(\theta_{1}, \theta_{2}\right)$,

$\alpha_{1}=\frac{1}{x_{1}^{2}}, \quad \alpha_{2}=-\frac{x_{4}}{x_{1}}, \quad \alpha_{3}=\frac{\beta\left(x_{2} x_{6}-x_{3} x_{5}\right)+K}{\alpha x_{1}^{2}}$,

$\alpha_{4}=m_{\mathrm{sat}} x_{1}\left[(1-\mu) \frac{x_{2}-\mu x_{1}}{r_{13}^{3}}+\mu \frac{x_{2}+(1-\mu) x_{1}}{r_{23}^{3}}\right]$,

$\alpha_{5}=-m_{\mathrm{sat}} x_{1} x_{3}\left[\frac{(1-\mu)}{r_{13}^{3}}+\frac{\mu}{r_{23}^{3}}\right]$,

$\alpha_{6}=\frac{1}{x_{1}}, \quad \alpha_{7}=\frac{x_{2}}{x_{1}}, \quad \alpha_{8}=-\frac{x_{3}}{x_{1}}$,

where $x_{j} \equiv x_{j}\left(\theta_{1}, \theta_{2}\right)$ denote the coordinates of $X\left(\theta_{1}, \theta_{2}\right), \alpha=$ $\mu(1-\mu), \beta=m_{\text {sat }} /\left(1+m_{\text {sat }}\right), r_{13}^{2}=\left(x_{2}-\mu x_{1}\right)^{2}+x_{3}^{2}, r_{23}^{2}=$ $\left(x_{2}+(1-\mu) x_{1}\right)^{2}+x_{3}^{2}$ and $K$ is the angular momentum of the system.

Hence, it is easy to obtain a tabulation of the functions $\left(\alpha_{i}\right)_{i=1 \div 8}$ on the mesh (13). Due to the parity properties of our solution of the Three-Body Problem, these functions can be expanded as

$\alpha_{i}\left(\theta_{1}, \theta_{2}\right)=\sum_{k \in \mathbb{Z}^{2}} \alpha_{i k}^{+} \cos \langle k, \theta\rangle, \quad i=1,3,4,6,7$,

$\alpha_{i}\left(\theta_{1}, \theta_{2}\right)=\sum_{k \in \mathbb{Z}^{2}} \alpha_{i k}^{+} \sin \langle k, \theta\rangle, \quad i=2,5,8$,

and the coefficients $\alpha_{i k}^{+}$can be obtained by means of a standard Fourier transform.
We have to select a concrete orbit on the torus $X\left(\theta_{1}, \theta_{2}\right)$. In other words, we have to write the angles $\theta_{1}(t)$ and $\theta_{2}(t)$ depending on the adimensional time as $\theta_{j}(t)=\omega_{j} t+\theta_{j}^{(0)}, j=1,2$, and select the values of the two phases, $\theta_{j}^{(0)}$. Note that both phases are zero if we choose $t=0$ when Sun, Jupiter and Saturn are on a collinear configuration, with the Sun at one side.

Finally, it is not difficult to write the Hamiltonian of the Bianular problem as:

$$
\begin{aligned}
H_{\mathrm{BAP}}= & \frac{1}{2} \alpha_{1}(t)\left(p_{x}^{2}+p_{y}^{2}+p_{z}^{2}\right)+\alpha_{2}(t)\left(x p_{x}+y p_{y}+z p_{z}\right) \\
& +\alpha_{3}(t)\left(y p_{x}-x p_{y}\right) \alpha_{4}(t) x+\alpha_{5}(t) y \\
& -\alpha_{6}(t)\left[\frac{1-\mu}{q_{S}}+\frac{\mu}{q_{J}}+\frac{m_{\text {sat }}}{q_{\mathrm{sat}}}\right]
\end{aligned}
$$

where $q_{S}^{2}=(x-\mu)^{2}+y^{2}+z^{2}, q_{J}^{2}=(x-\mu+1)^{2}+y^{2}+z^{2}$ and $q_{\mathrm{sat}}^{2}=\left(x-\alpha_{7}(t)\right)^{2}+\left(y-\alpha_{8}(t)\right)^{2}+z^{2}$. The concrete values for the coefficients of the quasi-periodic functions $\left(\alpha_{i}\right)_{i=1 \div 8}$ can be obtained from http://www.maia.ub.es/dsg/, in the preprints section.

\subsection{The Tricircular coherent problem}

In the reduced SJSU four body problem (5), the $\left(\alpha_{i}\right)_{i=1 \div 10}$ functions can be obtained from the following formulae:

$$
\begin{aligned}
\alpha_{1}= & \frac{1}{x_{1}^{2}}, \quad \alpha_{2}=-\frac{x_{6}}{x_{1}}, \\
\alpha_{3}= & \frac{\beta\left(x_{2} x_{8}-x_{3} x_{7}\right)+\gamma\left(x_{4} x_{10}-x_{5} x_{9}\right)+K}{\alpha x_{1}^{2}}, \\
\alpha_{4}= & m_{\mathrm{sat}} x_{1}\left[(1-\mu) \frac{x_{2}-\mu x_{1}}{r_{13}^{3}}+\mu \frac{x_{2}+(1-\mu) x_{1}}{r_{23}^{3}}\right] \\
& +m_{\mathrm{ura}}(1+\beta)(1-\mu) x_{1} \frac{x_{4}+\beta x_{2}-\mu x_{1}}{r_{14}^{3}} \\
& +m_{\mathrm{ura}}(1+\beta) \mu x_{1} \frac{x_{4}+\beta x_{2}+(1-\mu) x_{1}}{r_{24}^{3}} \\
& +m_{\mathrm{ura}} \beta m_{\mathrm{sat}} x_{1} \frac{x_{4}-(1-\beta) x_{2}}{r_{34}^{3}}, \\
\alpha_{5}= & -m_{\mathrm{sat}} x_{1} x_{3}\left[\frac{(1-\mu)}{r_{13}^{3}}+\frac{\mu}{r_{23}^{3}}\right] \\
& -m_{\mathrm{ura}}(1+\beta) x_{1}\left[(1-\mu) \frac{x_{5}+\beta x_{3}}{r_{14}^{3}}+\mu \frac{x_{5}+\beta x_{3}}{r_{24}^{3}}\right] \\
& +m_{\mathrm{ura}} \beta m_{\mathrm{sat}} x_{1} \frac{x_{5}-(1-\beta) x_{3}}{r_{34}^{3}}, \\
& 1
\end{aligned}
$$

$\alpha_{6}=\frac{1}{x_{1}}, \quad \alpha_{7}=\frac{x_{2}}{x_{1}}, \quad \alpha_{8}=-\frac{x_{3}}{x_{1}}$,

$\alpha_{9}=\frac{x_{4}+\beta x_{2}}{x_{1}}, \quad \alpha_{10}=-\frac{x_{5}+\beta x_{3}}{x_{1}}$,

where, as usual, $\alpha=\mu(1-\mu), \beta=m_{\mathrm{sat}} /\left(1+m_{\mathrm{sat}}\right), \gamma=(1+$ $\left.m_{\text {sat }}\right) m_{\text {ura }} /\left(1+m_{\text {sat }}+m_{\text {ura }}\right), r_{13}^{2}=\left(x_{2}-\mu x_{1}\right)^{2}+x_{3}^{2}, r_{23}^{2}=\left(x_{2}+(1-\right.$ p) $\left.x_{1}\right)^{2}+x_{3}^{2}, r_{14}^{2}=\left(x_{5}+\beta x_{3}\right)^{2}+\left(x_{4}+\beta x_{2}-\mu x_{1}\right)^{2}, r_{24}^{2}=\left(x_{5}+\beta x_{3}\right)^{2}+$ $\left(x_{4}+\beta x_{2}+(1-\mu) x_{1}\right)^{2}, r_{34}^{2}=\left(x_{5}-(1-\beta) x_{3}\right)^{2}+\left(x_{4}-(1-\beta) x_{2}\right)^{2}$ and $K$ is the angular momentum of the system. 
In the same way as in the Bianular Problem, it is possible to write them as real Fourier expansions in the following way:

$$
\begin{array}{ll}
\alpha_{i}\left(\theta_{1}, \theta_{2}\right)=\sum_{k \in \mathbb{Z}^{2}} \alpha_{i k}^{+} \cos \langle k, \theta\rangle, & i=1,3,4,6,7,9 \\
\alpha_{i}\left(\theta_{1}, \theta_{2}\right)=\sum_{k \in \mathbb{Z}^{2}} \alpha_{i k}^{+} \sin \langle k, \theta\rangle, & i=2,5,8,10
\end{array}
$$

where the angles are given by $\theta_{1}=\omega_{\text {sat }} t$ and $\theta_{2}=\omega_{\text {ura }} t$. The concrete values of the Fourier coefficients can be obtained from http://www. maia.ub.es/dsg/, in the preprints section.

Finally, the Hamiltonian of the Tricircular Coherent Problem is written as

$$
\begin{aligned}
H_{\mathrm{TCCP}}= & \frac{1}{2} \alpha_{1}(t)\left(p_{x}^{2}+p_{y}^{2}+p_{z}^{2}\right)+\alpha_{2}(t)\left(x p_{x}+y p_{y}+z p_{z}\right) \\
& +\alpha_{3}(t)\left(y p_{x}-x p_{y}\right)+\alpha_{4}(t) x+\alpha_{5}(t) y \\
& -\alpha_{6}(t)\left[\frac{1-\mu}{q_{S}}+\frac{\mu}{q_{J}}+\frac{m_{\text {sat }}}{q_{\mathrm{sat}}}+\frac{m_{\mathrm{ura}}}{q_{\text {ura }}}\right],
\end{aligned}
$$

where $q_{S}^{2}=(x-\mu)^{2}+y^{2}+z^{2}, q_{J}^{2}=(x-\mu+1)^{2}+y^{2}+z^{2}$, $q_{\mathrm{sat}}^{2}=\left(x-\alpha_{7}(t)\right)^{2}+\left(y-\alpha_{8}(t)\right)^{2}+z^{2}$ and $q_{\text {ura }}^{2}=\left(x-\alpha_{9}(t)\right)^{2}+$ $\left(y-\alpha_{10}(t)\right)^{2}+z^{2}$.

\subsection{Tests}

To check the computations of the $\alpha_{i}$ functions of the BAP and TCCP Hamiltonians, as well as the changes of variables, we have integrated some different test particles (with initial conditions selected at random) in the fields coming from $H_{\mathrm{BAP}}$ and $H_{\mathrm{TCC}}$. Then, by means of the previous changes of variables, we have sent these orbits to the initial Jacobi coordinates of the restricted $N$-body problems to check that they correspond to orbits of this system (this is again checked by a numerical integration of the $N$-body problem). The accuracy agrees with the order of truncation of the Fourier coefficients.

\section{Conclusions}

In this paper, we have considered the development of two models for the motion of an asteroid in the Solar system. These models are constructed as improvements of the BCCP model which is an improvement of the RTBP.

The first of these models is based on computing a quasiperiodic solution of the general planar Sun-Jupiter-Saturn system. In this solution, Jupiter moves on a nearly Keplerian orbit whose eccentricity is the real one. The second model is based on the computation of a quasi-periodic solution of the Sun-Jupiter-Saturn-Uranus problem. This is the simplest planetary solution for these bodies, so that the obtained model can be seen as the natural extension of the RTBP to include four primaries. In both models, the motion of the asteroid is finally described by a quasi-periodic time-dependent perturbation of the RTBP.

These models are still too close to the RTBP to be an alternative to the Outer Solar System model but, on the other hand, they are written in an explicit way. In this sense, they should be considered as improvements of the RTBP towards real situations. Moreover, the explicit character of the models greatly simplifies the use of semi-analytical techniques, like normal forms. This is the topic of a forthcoming work.

Acknowledgements. We thank the comments of R. Dvorak on a preliminary version of this paper. Work supported by the MCyT/FEDER Grant BFM2003-07521-C02-01, the CIRIT grant 2001SGR-70 and DURSI.

\section{References}

Broer, H., Hanßmann, H., Jorba, A., Villanueva, J., \& Wagener, F. 2003, Nonlinearity, 16, 1751

Castellà, E., \& Jorba, À. 2000, Celest. Mech., 76, 35

Celletti, A., \& Giorgilli, A. 1991, Celest. Mech., 50, 31

Cronin, J., Richards, P., \& Russell, L. 1964, Icarus, 3, 423

Gabern, F., \& Jorba, A. 2001, Discrete Contin. Dynam. Systems Series B, 1, 143

Gabern, F., Jorba, A., \& Robutel, P. 2003, preprint

Giorgilli, A., Delshams, A., Fontich, E., Galgani, L., \& Simó, C. 1989, J. Differential Equations, 77, 167

Giorgilli, A., \& Skokos, C. 1997, A\&A, 317, 254

Gómez, G., Jorba, À., Simó, C., \& Masdemont, J. 2001a, World Scientific Monograph Series in Mathematics, 4

Gómez, G., Jorba, À., Simó, C., \& Masdemont, J. 2001b, World Scientific Monograph Series in Mathematics, 5

Gómez, G., Llibre, J., Martínez, R., \& Simó, C. 2001c, World Scientific Monograph Series in Mathematics, 2

Gómez, G., Simó, C., Llibre, J., \& Martínez, R. 2001d, World Scientific Monograph Series in Mathematics, 3

Gómez, G., Masdemont, J., \& Mondelo, J. 2002, A\&A, 390, 733

Hadjidemetriou, J. D. 1980, Celest. Mech., 21, 63

Jorba, À. 1999, Exp. Math., 8, 155

Jorba, À., \& Masdemont, J. 1999, Phys. D, 132, 189

Jorba, À., \& Villanueva, J. 1997, Nonlinearity, 10, 783

Jorba, À., \& Villanueva, J. 2001, in Progr. Math., 202, European Congress of Mathematics, II, Barcelona, 2000 (Basel: Birkhäuser), 557

Laskar, J. 1989, Les variables de Poincaré et le developpement de la fonction perturbatrice, Notes scientifiques et techniques du Bureau des Longitudes S026, Groupe de travail sur la lecture des Méthodes nouvelles de la Mécanique Céleste

Levison, H., Shoemaker, E., \& Shoemaker, C. 1997, Nature, 385, 42

Milani, A. 1993, Celest. Mech., 57, 59

Pilat-Lohinger, E., Dvorak, R., \& Burger, C. 1999, Celest. Mech., 73, 117

Robutel, P., \& Laskar, J. 2001, Icarus, 152, 4

Schubart, J., \& Bien, R. 1986, in Asteroids, Comets, Meteors II, 153

Simó, C. 1989, Mem. Real Acad. Cienc. Artes Barcelona, 48, 303

Skokos, C., \& Dokoumetzidis, A. 2000, A\&A, 367, 729

Szebehely, V. 1967, Theory of Orbits (Academic Press)

Tsiganis, K., Dvorak, R., \& Pilat-Lohinger, E. 2000, A\&A, 354, 1091

Whittaker, E. 1952, A treatise on the analytical dynamics of particles and rigid bodies: with an introduction to the problem of three bodies. (Cambridge: Cambridge Univ. Press) 